\title{
EVOLUCIÓN TEMPORAL DEL DIFERENCIAL DE GÉNERO EN LA COMUNIDAD AUTÓNOMA DE ANDALUCÍA. COMPARACIÓN CON OTRAS COMUNIDADES ESPAÑOLAS
}

\author{
María José VÁZQUEZ CUETO*
}

\section{INTRODUCCIÓN}

En 1999, la Unión Europea, en el marco de la Agenda 2000, reformó algunas de sus políticas básicas, y en concreto su política regional. Para utilizar el dinero de los Fondos Estructurales con la mayor eficiencia posible. Las políticas activas de lucha contra eI desempleo y, dentro de ellas, las medidas positivas que mejoran la participación de las mujeres en eI mercado laboral, constituyen una de las grandes prioridades de la aplicación de estos Fondos en los años 2000-2006.

El 1 de Mayo de 1999 entra en vigor el Tratado de Amsterdam, aprobado en 1997, del que surge el Plan del Objetivo 3 para España, periodo 2000-2006, que recoge las actuaciones a favor de los Recursos Humanos objeto de cofinanciación por el Fondo Social Europeo. Este Plan establece el marco de referencia para la política de desarrollo de los recursos humanos en todo el territorio español para el período 2000-2006. En este sentido, y para distribuir los costes previstos de las distintas actuaciones que se emprendan, se hace una clasificación de las distintas Comunidades Autónomas en función de su renta per cápita ${ }^{1}$. (Cuadro I).

Cantabria deja de ser objetivo 1 por ser su renta per cápita de 1994, 1995 y 1996 superior al 75\% de la renta per cápita media comunitaria.

A pesar de esta declaración de intenciones con respecto a la posición femenina en el mercado laboral, es obvio, y la simple observación de datos absolutos y

* Catedrática de Economía Aplicada. Universidad de Sevilla.

1. Son elegidas como objetivo 1 aquellas regiones cuya renta per cápita no supere el $75 \%$ de la media comunitaria. Las restantes son elegidas como objetivo 3. 
relativos lo confirma, que éste no trata por igual a hombres y mujeres, en perjuicio de las segundas. Esta situación, aún habiendo sido establecidas medidas correctoras, está aún lejos de corregirse por completo.

Este trabajo trata de mostrar cómo está reaccionando el mercado de trabajo andaluz ante estas medidas, analizando la evolución experimentada por el diferencial de género en el empleo y en el paro entre los años 1993 a 1996 y 1996 a 1999. Esta evolución se compara con la seguida por las otras Comunidades que son consideradas como "Regiones objetivo 1" por la Unión Europea.

\section{CUADRO I}

Objetivo 1

Comunidades Autónomas

Andalucía, Asturias, Canarias, Castilla-La Mancha, Castilla-León, Valencia, Galicia, Extremadura, Murcia, Ceuta y Melilla.

Objetivo 2

Comunidades Autónomas

Aragón, Baleares, Cataluña, Madrid, Navarra, País Vasco, La Rioja.

Fuente: Panorama. Elaboración propia.

La técnica de análisis empleada está apoyada en la representación de la situación mediante gráficos radiales. La oportunidad de los gráficos, junto con las ventajas e inconvenientes de los mismos, serán, también, objeto de estudio.

El trabajo se estructura de la siguiente forma: Tras esta introducción se dedica el apartado 2 a la presentación de la metodología que se utilizará a lo largo del desarrollo del mismo. En el apartado 3 se definen los indicadores que van a utilizarse. El cuarto apartado aplica la metodología y presenta los resultados. El quinto y último apartado del análisis extrae las conclusiones del mismo. En el sexto y último apartado del trabajo se recoge la bibliografía.

\section{METODOLOGÍA}

Para realizar un análisis del comportamiento del gap de género en el empleo y en el paro en la Comunidad Autónoma de Andalucía, hemos elegido la técnica de representación de datos en un gráfico radial.

Dos son las aplicaciones de esta técnica. Podemos representar en un mismo gráfico la situación en dos o más momentos del tiempo. Esto nos indicará cómo va evolucionando. O bien podemos elegir un mismo periodo de tiempo y representar en el gráfico la situación, en dicho momento, de varios entes. Esto nos permitirá establecer comparaciones entre los mismos. 
Entre las ventajas que ofrecen los análisis apoyados en estos gráficos, consideramos como una de las más importantes el hecho de que los mismos nos permiten manejar simultáneamente varias variables, lo que da una mejor aproximación a la realidad de la situación que estamos analizando. Además, esta forma de representación de los datos nos ofrece una simple y rápida conclusión acerca de la mejor o peor posición que ocupamos con respecto a la situación de partida. Obviamente, cuantos más ejes tenga el gráfico mejor será la representación de la realidad, pero a la vez se irá perdiendo el poder de visualización de la mejora. Debemos, pues, combinar ambos objetivos de nuestro análisis, eligiendo un número apropiado de ejes como para tener una buena aproximación de la situación sin perder la interpretación visual de los resultados.

Entre las desventajas de esta técnica consideramos como más significativa el hecho de que no nos proporciona ninguna explicación acerca de la evolución experimentada. Esta deberá ser analizada con otras herramientas.

Una vez que hemos fijado el número de ejes que va a tener nuestro gráfico, en función de lo que vamos a medir, de los datos que disponemos y de una combinación óptima entre una buena representación de la realidad y una rápida visualización de la situación, habrá que proceder a calcular los indicadores definidos y a homogeneizar los mismos, pues el gráfico perderá significación si presenta unos valores en los ejes que no guardan relación entre los mismos. La estandarización de los datos es, pues, de obligado cumplimiento y va a condicionar, al igual que la elección de los factores, los resultados que se extraigan.

Existen varias formas de estandarización, en nuestro trabajo optamos por hacerlo siguiendo direcciones de mejora. Para el estudio de la mejora experimentada por un mismo ente a lo largo del tiempo, elegimos tres años t, t-1 y t-2. En primer lugar analizamos la mejora de $\mathrm{t}-1$ a t dando el valor de 1 al mejor valor del indicador en el año $t$ y el valor de 0 al peor valor del indicador entre los años t-1 y t. A continuación repetimos el proceso para la mejora de t-2 a t-1. De esta forma podremos representar en un mismo gráfico dos líneas, una correspondiente a la mejora experimentada en t-1 y otra correspondiente a la mejora experimentada en t, así podremos ver la evolución de la mejora experimentada por un mismo ente a lo largo del tiempo. (Gráfico 1).

Tratando de comparar la mejora experimentada del año t-1 al año $t$, por distintos entes, para cada indicador asignamos un valor de 1 al mejor valor encontrado en el año $t$, y un valor de 0 al peor valor encontrado entre los años $t-1$ y t. De esta forma todos los valores van a ser positivos. Un valor de 0,5 significará que este indicador ha mejorado para este ente la mitad de lo que ha mejorado en el que más lo ha hecho. Un valor mayor que 1 nos avisará de que este indicador ha empeorado a lo largo del tiempo para dicho ente. (Gráfico 2).

Por otra parte el mismo gráfico nos puede servir para elaborar un indicador conjunto de la mejora global en los objetivos marcados. El área de la figura for- 
mada por los ejes, calculada matemáticamente, puede ser utilizada para comparar la mejora global experimentada por los distintos entes o por un mismo ente en distintos periodos de tiempo.

\section{INDICADORES}

Dado que nuestro objetivo es analizar si se ha reducido el gap de género en el mercado laboral andaluz, son variables de obligada inclusión el diferencial de género en el empleo y el diferencial de género en el paro. Puesto que estas

\section{GRÁFICO 1}

EVOLUCIÓN TEMPORAL

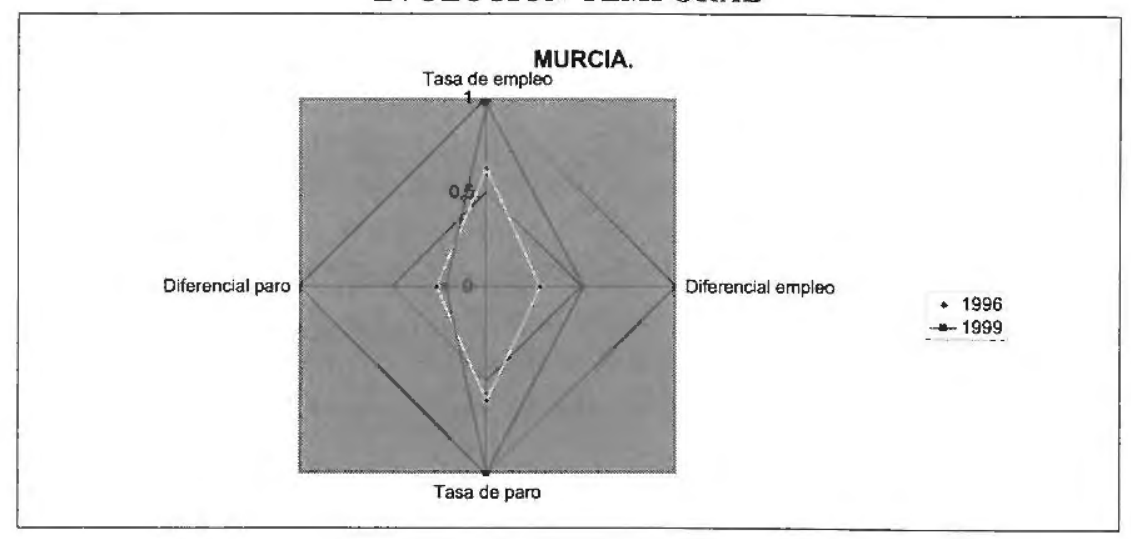

GRÁFICO 2

ANÁLISIS COMPARATIVO

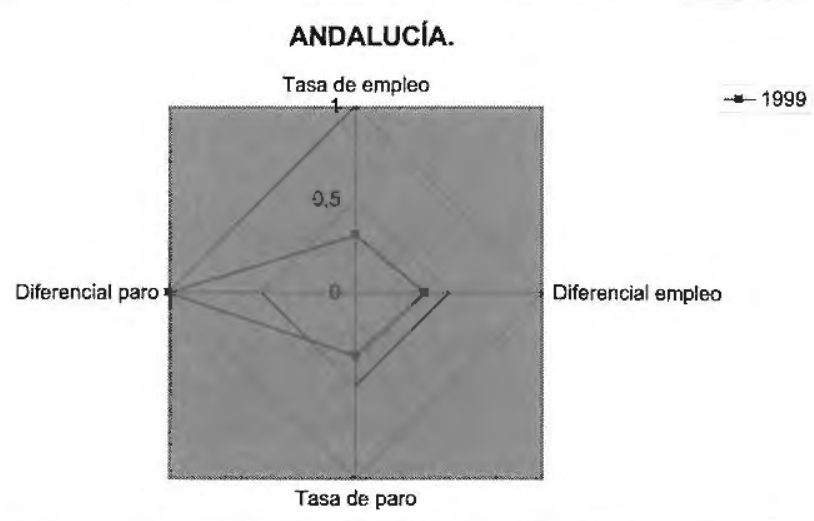


variables pueden verse condicionados por un distinto comportamiento en el empleo y en el paro, optamos, para representar la situación, por un gráfico de cuatro ejes, donde representaremos el empleo, el diferencial en el empleo, el paro y el diferencial en el paro.

La medición de estas variables la realizaremos a través de los siguientes indicadores:

- Para la variable empleo utilizaremos la TASA DE EMPLEO, definida como el cociente entre el número de ocupados y la población mayor de 16 años.

- Para el diferencial de empleo utilizaremos el COCIENTE ENTRE LA TASA DE EMPLEO FEMENINO Y LA TASA DE EMPLEO MASCULINO, definida cada una de ellas de la misma forma que la tasa de empleo pero relativa al colectivo en cuestión.

- Para la variable paro utilizaremos la TASA DE PARO, definida como el cociente entre el número de parados y la población activa.

- Para el diferencial de paro utilizaremos el COCIENTE ENTRE LA TASA DE PARO MASCULINO Y LA TASA DE PARO FEMENINO, definida cada una de ellas de la misma forma que la tasa de paro pero relativa al colectivo en cuestión.

Como el objetivo del trabajo es doble: analizar la evolución experimentada por Andalucía y compararla con la seguida por otras Comunidades, utilizaremos los valores de los indicadores en los años 1993, 1996 y 1999 de nuestra Región y de las Comunidades que constituyen el "objetivo 1".

Con los datos ofrecidos por el Instituto Nacional de Estadística en las Encuestas de Población Activa correspondientes a los segundos trimestres de los años 1993, 1996 y 1999, elaboramos el cuadro 2 de indicadores.

\section{RESULTADOS}

\subsection{Evolución del diferencial de género en la Comunidad Autónoma de Andalucía}

En el siguiente gráfico mostramos la mejora experimentada de 1996 a I999, con respecto a la situación que estamos analizando, en la Comunidad Autónoma de Andalucía. (Gráfico 3).

La Comunidad Autónoma de Andalucía ocupa la primera posición en cuanto a la mejora experimentada por el diferencial de paro de 1996 a 1999. El empleo, sin embargo, no ha respondido como en el resto de las Comunidades con las que es- 
tamos comparando a la nuestra. Aún habiendo mejorado la tasa de empleo y el diferencial de empleo, el primero no lo ha hecho ni el $30 \%$ de lo que ha mejorado la mejor Comunidad. En cuanto al segundo, la situación es ligeramente mejor, alcanzando una mejora del $36 \%$ de la experimentada por la mejor Comunidad. De la misma forma la tasa de paro sigue siendo uno de los mayores problemas en nuestra Comunidad. Aún habiendo mejorado en 1999, esta mejora no llega ni al $30 \%$ de la producida en las otras Comunidades.

\section{CUADRO II \\ INDICADORES}

\begin{tabular}{|c|c|c|c|c|c|c|c|c|c|c|c|c|}
\hline \multirow{2}{*}{ CCAA } & \multicolumn{3}{|c|}{ Tasa de Empleo } & \multicolumn{3}{|c|}{ Diferencial Empleo } & \multicolumn{3}{|c|}{ Tasa de Paro } & \multicolumn{3}{|c|}{ Diferencial Paro } \\
\hline & 1999 & 1996 & 1993 & 1999 & 1996 & 1993 & 1999 & 1996 & 1993 & 1999 & 1996 & 1993 \\
\hline Andalucía & 35,97 & 32,56 & 31,95 & 0,45 & 0,46 & 0,44 & 26,06 & 32,39 & 32,3 & 0,53 & 0,66 & 0,78 \\
\hline Asturias & 34,84 & 33,56 & 35,26 & 0,52 & 0,46 & 0,49 & 17,65 & 22,22 & 20,4 & 0,50 & 0,61 & 0,59 \\
\hline Cantabria & 38,11 & 34,49 & 37,83 & 0,43 & 0,47 & 0,44 & 15,19 & 24,52 & 20,0 & 0,41 & 0,57 & 0,58 \\
\hline Castilla-León & 39,68 & 36,90 & 36,53 & 0,48 & 0,44 & 0,42 & 15,19 & 20,22 & 20,0 & 0,35 & 0,46 & 0,45 \\
\hline Cast.-La Mancha & 40,52 & 35,93 & 36,15 & 0,42 & 0,38 & 0,38 & 15,13 & 19,97 & 19,5 & 0,38 & 0,47 & 0,53 \\
\hline Valenciana & 43,83 & 40,47 & 39,34 & 0,53 & 0,50 & 0,48 & 13,88 & 21,47 & 23,8 & 0,44 & 0,58 & 0,62 \\
\hline Extremadura & 35,57 & 32,47 & 32,11 & 0,42 & 0,42 & 0,36 & 24,75 & 30,18 & 30,3 & 0,50 & 0,73 & 0,58 \\
\hline Galicia & 40,27 & 39,76 & 41,45 & 0,57 & 0,58 & 0,59 & 16,29 & 18,96 & 17,6 & 0,48 & 0,65 & 0,74 \\
\hline Murcia & 43,46 & 37,31 & 38,65 & 0,48 & 0,44 & 0,46 & 14,03 & 24,24 & 24,5 & 0,39 & 0,47 & 0,65 \\
\hline Ceuta y Melilla & 39,63 & 36,10 & 33,66 & 0,40 & 0,70 & 0,34 & 24,60 & 27,01 & 22,70 & 0,47 & 0,59 & 0,40 \\
\hline
\end{tabular}

CUADRO III

DATOS ESTANDARIZADOS. PERÍODO 1993-1996

\begin{tabular}{lllllllll}
\hline & \multicolumn{2}{c}{ Tasa de Empleo } & \multicolumn{2}{c}{ Diferencial Empleo } & \multicolumn{2}{c}{ Tasa de Paro } & \multicolumn{2}{c}{ Diferencial Paro } \\
\cline { 2 - 9 } & 1996 & 1993 & 1996 & 1993 & 1996 & 1993 & 1996 & 1993 \\
\hline Andalucía & 0,0716 & 0 & 0,319 & 0,26982 & 0 & 0,006 & 1 & 1,4615 \\
Asturias & 0,189 & 0,3885 & 0,3453 & 0,40426 & 0,75726 & 0,8935 & 0,80769 & 0,7308 \\
Cantabria & 0,2981 & 0,6901 & 0,3743 & 0,28108 & 0,586 & 0,9255 & 0,65385 & 0,6923 \\
Castilla-León & 0,581 & 0,5376 & 0,2667 & 0,21578 & 0,90618 & 0,9226 & 0,23077 & 0,1923 \\
Cast.-La Mancha & 0,4671 & 0,493 & 0,1016 & 0,07173 & 0,9248 & 0,9598 & 0,26923 & 0,5 \\
Valenciana & 1 & 0,8674 & 0,4386 & 0,36668 & 0,8131 & 0,6374 & 0,69231 & 0,8462 \\
Extremadura & 0,061 & 0,0188 & 0,2099 & 0,06289 & 0,16456 & 0,1564 & 0,88462 & 0,6923 \\
Galicia & 0,9167 & 1,115 & 0,6715 & 0,70301 & 1 & 1,105 & 0,96154 & 1,3077 \\
Murcia & 0,6291 & 0,7864 & 0,283 & 0,33201 & 0,60685 & 0,5867 & 0,26923 & 0,9615 \\
Ceuta y Melilla & 0,4871 & 0,2007 & 1 & 0 & 0,4006 & 0,7238 & 0,73077 & 0 \\
\hline
\end{tabular}


CUADRO IV

DATOS ESTANDARIZADOS. PERÍODO 1996-1999

\begin{tabular}{llllllllll}
\hline & \multicolumn{2}{c}{ Tasa de Empleo } & \multicolumn{2}{c}{ Diferencial Empleo } & \multicolumn{2}{c}{ Tasa de Paro } & \multicolumn{2}{c}{ Diferencial Paro } \\
\cline { 2 - 10 } & 1999 & 1996 & 1999 & 1996 & 1999 & 1996 & 1999 & 1996 \\
\hline Andalucía & 0,3081 & 0,0079 & 0,3658 & 0,40058 & 0,34198 & 0 & 1 & 1,7222 \\
Asturias & 0,2086 & 0,096 & 0,7229 & 0,44902 & 0,79633 & 0,5494 & 0,83333 & 1,4444 \\
Cantabria & 0,4965 & 0,1778 & 0,2458 & 0,50244 & 0,92923 & 0,4252 & 0,33333 & 1,2222 \\
Castilla-León & 0,6347 & 0,39 & 0,537 & 0,30414 & 0,92923 & 0,6575 & 0 & 0,611 \\
Cast.-La Mancha & 0,7086 & 0,3046 & 0,2295 & 0 & 0,93247 & 0,671 & 0,16667 & 0,6667 \\
Valenciana & 1 & 0,7042 & 0,7988 & 0,62098 & 1 & 0,59 & 0,5 & 1,2778 \\
Extremadura & 0,2729 & 0 & 0,2121 & 0,19946 & 0,41275 & 0,1194 & 0,83333 & 1,556 \\
Galicia & 0,6866 & 0,6417 & 1 & 1,05008 & 0,8698 & 0,7256 & 0,72222 & 1,6667 \\
Murcia & 0,9674 & 0,4261 & 0,5053 & 0,33415 & 0,9919 & 0,4403 & 0,22222 & 0,6667 \\
Ceuta y Melilla & 0,6303 & 0,3195 & 0,1312 & 1,65535 & 0,42085 & 0,2907 & 0,66667 & 1,3333 \\
\hline
\end{tabular}

GRÁFICO 3

MEJORA EXPERIMENTADA POR LA COMUNIDAD DE ANDALUCÍA, DE 1996 A 1999. (Elaboración propia)

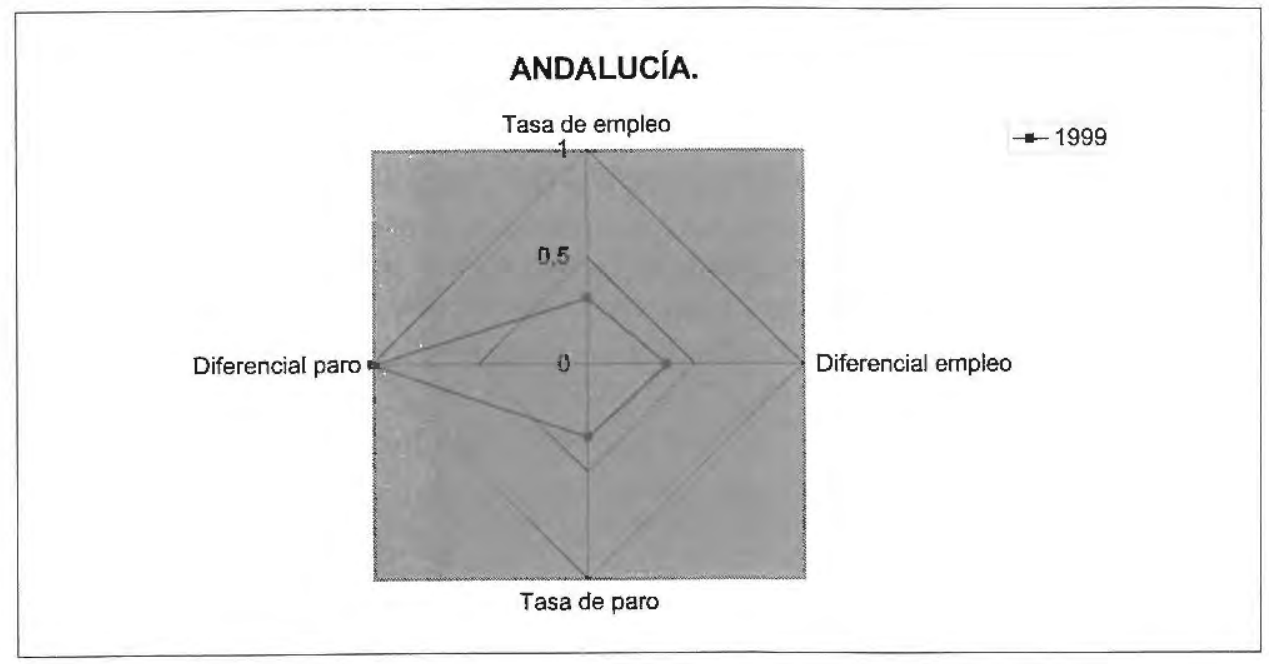

Estas comparaciones podrán visualizarse de forma completa en el gráfico 4. Tal como están representadas observamos que ninguna de ellas domina claramente, en el sentido que mejore en todos los ejes, a las otras. Así Andalucía muestra un magnífico comportamiento, siempre hablando en términos relativos, respecto 


\section{GRÁFICO 4}

MEJORA EXPERIMENTADA POR LAS COMUNIDADES AUTÓNOMAS DE 1996 A 1999. (Elaboración propia)

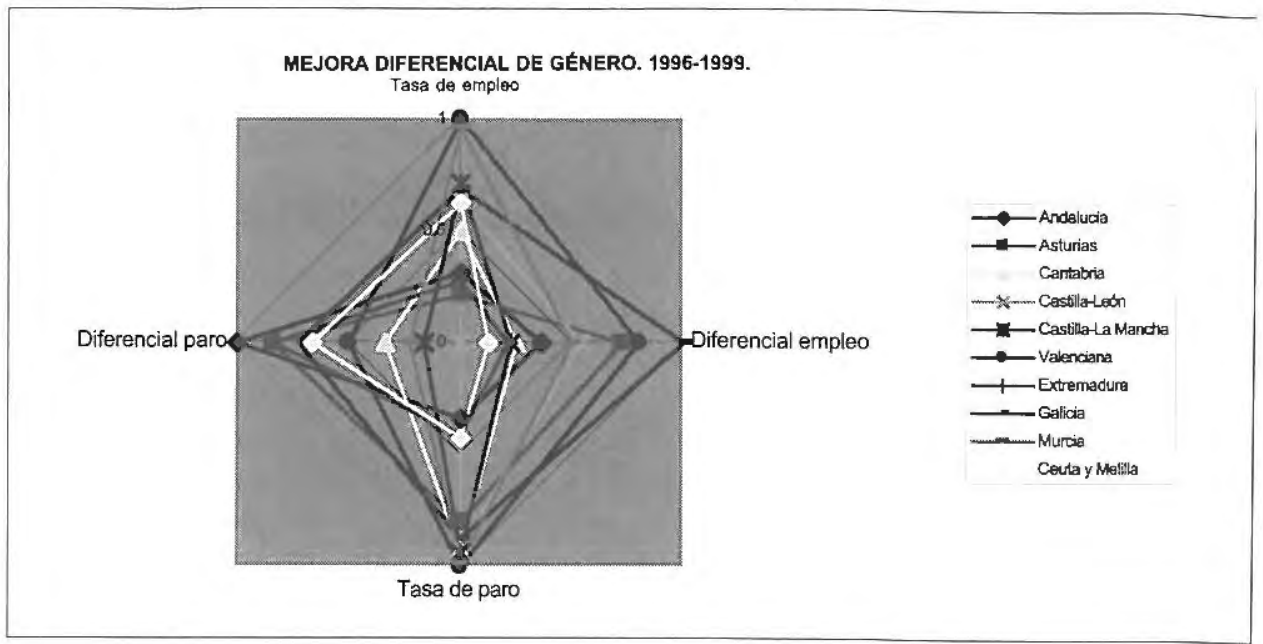

al diferencial en el paro, pero, la Comunidad Valenciana es la que muestra un mejor comportamiento en las tasas de empleo y paro, y la Comunidad Gallega en el diferencial de empleo.

La mejora global puede analizarse mediante el indicador conjunto, el cual nos permitirá, además, realizar comparaciones entre las Comunidades que estamos analizando. (Gráfico 5).

Este indicador conjunto nos permite ordenar a las Comunidades por orden de mayor a menor mejora experimentada, globalmente, entre 1996 y 1999.

\begin{tabular}{ll}
\hline CCAA & 1999 \\
\hline Galicia & 1,323749699 \\
Valenciana & 1,282791551 \\
Asturias & 0,77234863 \\
Murcia & 0,703954691 \\
Andalucía & 0,438468951 \\
Castilla-León & 0,414718965 \\
Ceuta y Melilla & 0,414157878 \\
Cantabria & 0,407738232 \\
Extremadura & 0,353997854 \\
Castilla-La Mancha & 0,321053334 \\
\hline
\end{tabular}


GRÁFICO 5

INDICADOR CONJUNTO. (Elaboración propia)

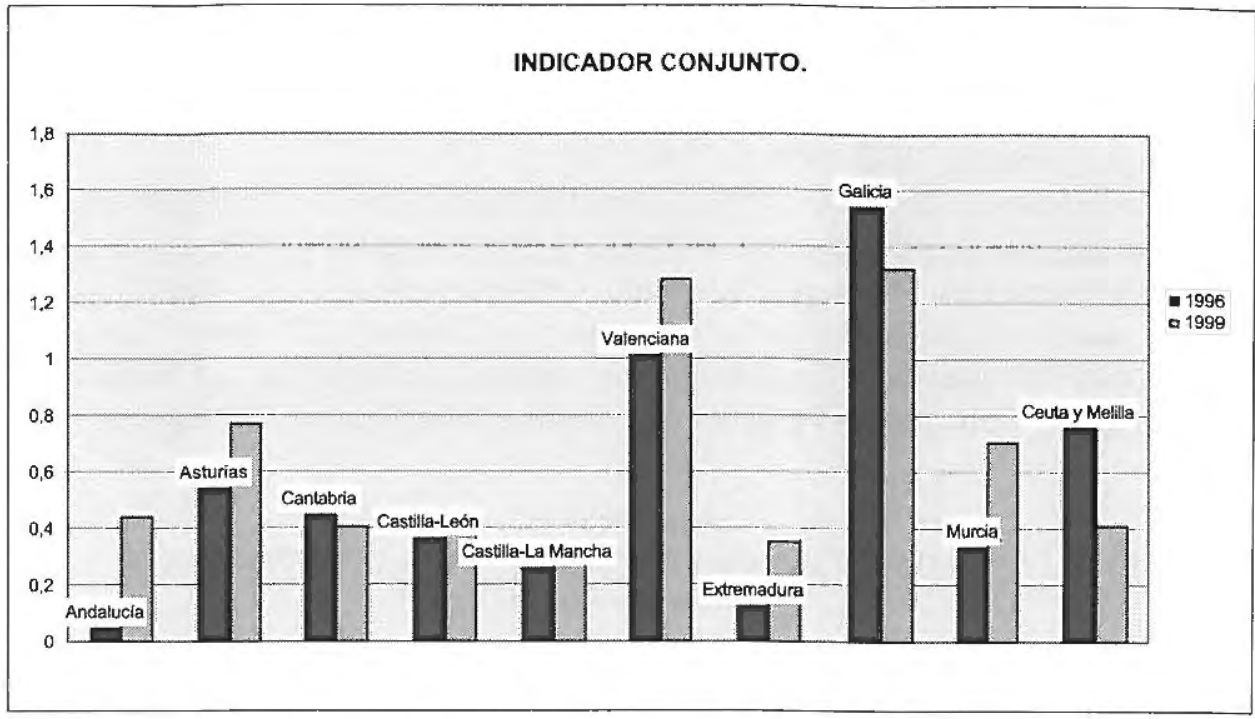

La Comunidad Autónoma de Galicia es, dentro del grupo de las regiones designadas por la Comunidad Europea como "Regiones objetivo 1", Ia que más ha mejorado su posición global respecto a los gaps de género en el empleo y en e1 paro, con un indicador global del 132,37\%. Muy cerca de ella está la Comunidad de Valenciana. Por el contrario Castilla-La Mancha y Extremadura son las que presentan peores posiciones. La Comunidad Autónoma de Andalucía ocupa la quinta de las diez Comunidades consideradas, esto se debe a lo que ya hemos comentado de una sólida mejora en el diferencial de paro que no se ha visto acompañada por el comportamiento en el empleo. Debemos hacer notar, también, la disparidad de comportamientos en las regiones que la Unión Europea ha englobado bajo el mismo epígrafe. Entre ellas, y en términos relativos, sus posiciones respecto al empleo, al paro, y a los gaps de género varían del $132,37 \%$ al $32,11 \%$, una horquilla de más de 100 puntos. De cualquier forma, teniendo en cuenta que el máximo valor que toma este indicador, tal como están homogeneizados los datos, es de 2, nos encontramos con que seis de ellas no ha alcanzado ni la mitad de lo que sería una completa mejora. 


\subsection{Evolución temporal de la mejora en el diferencial de género en la Comunidad Autónoma de Andalucía. Comparación con otras Comunidades}

En el gráfico 6 mostramos la evolución de la mejora de la Comunidad Andaluza en los periodos 1993-1996 a 1996-1999.

Observamos que de 1996 a 1999 el empleo evoluciona en esta región frente a las otras mejor que como lo hizo de 1993 a 1996. No así en el diferencial de género en el empleo, en el que prácticamente mantiene su posición. Es decir, de 1993 a 1996 el diferencial de género en el empleo evoluciona de la misma forma que de 1996 a 1999. Ambos indicadores no mejoran ni la mitad de lo que lo hacen en el resto de las Comunidades con las que estamos comparando a Andalucía.

\section{GRÁFICO 6}

EVOLUCIÓN DE LA MEJORA EXPERIMENTADA POR ANDALUCÍA.

(Elaboración propia)

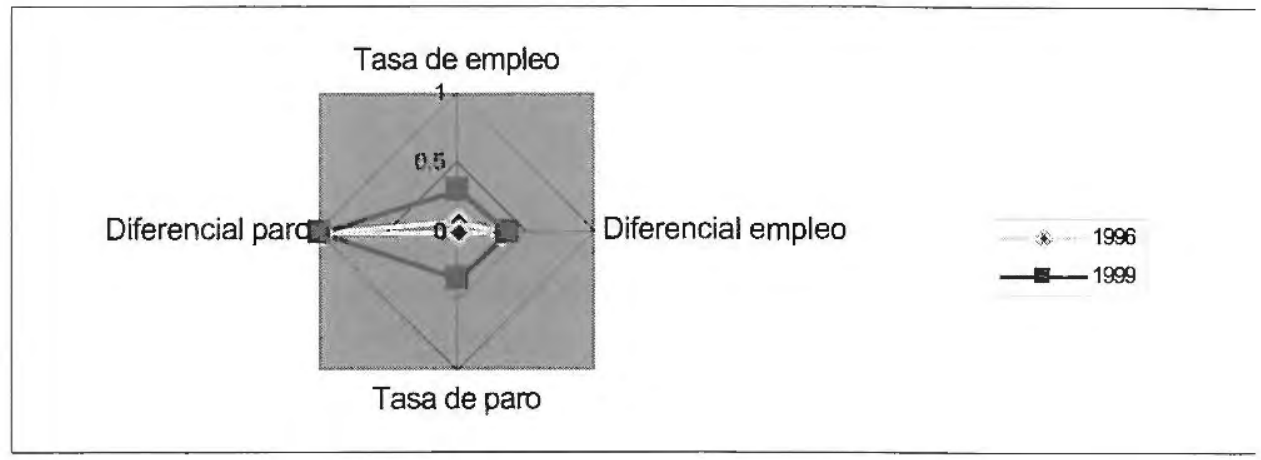

La posición que ocupa Andalucía respecto al paro es muy similar a la relativa al empleo. El diferencial de paro no se mueve, manteniendo la mayor de las mejoras que se producen en el resto de las Comunidades Autónomas. El paro mejora aunque no alcanza ni el 50\% de la mejora que experimenta en el resto de las Comunidades.

En los gráficos 7 a 15 se muestran las evoluciones seguidas por las otras Comunidades, comentándolas brevemente.

Mejora considerablemente el diferencial de empleo, mientras que permanecen inalterable el resto de los indicadores. (Gráfico 7).

Mejoran las tasas, sobre todo la del paro, mientras que empeoran los diferenciales, sobre todo el de paro. (Gráfico 8).

Las tasas de empleo y paro permanecen inalterables, mientras que mejora el diferencial de empleo y empeora el de paro. (Gráfico 9). 


\section{GRÁFICO 7}

EVOLUCIÓN DE LA MEJORA EXPERIMENTADA POR ASTURIAS.

(Elaboración propia)

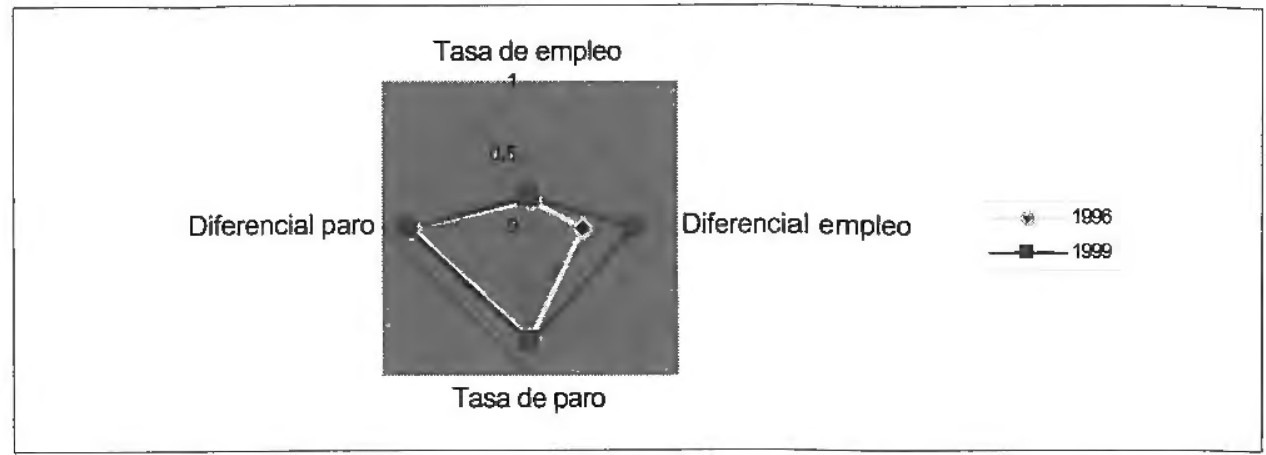

\section{GRÁFICO 8}

EVOLUCIÓN DE LA MEJORA EXPERIMENTADA POR CANTABRIA. (Elaboración propia)

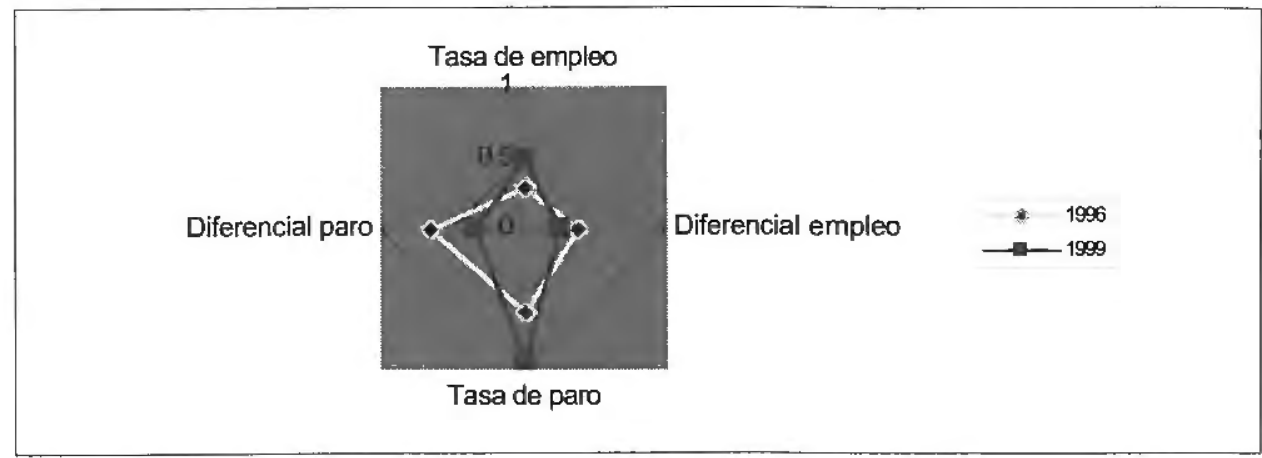

\section{GRÁFICO 9}

EVOLUCIÓN DE LA MEJORA EXPERIMENTADA POR CASTILLA-LEÓN. (Elaboración propia)

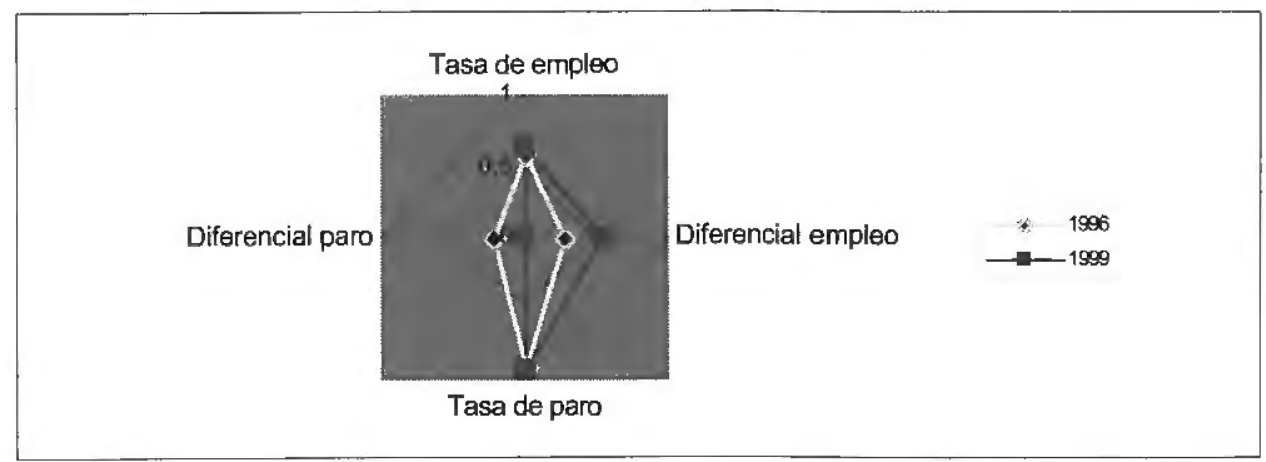


Mejoran levemente todos los indicadores salvo el diferencial de paro. (Gráfico 10)

$\mathrm{El}$ comportamiento del empleo y del paro son bien diferenciados. El primerc de ellos mejora el diferencial y permanece la tasa, mientras que el segundo mejo ra la tasa y empeora considerablemente el diferencial. (Gráfico 11).

Los diferenciales permanecen inalterables mientras que mejoran considerable. mente las tasas. (Gráfico 12).

Salvo el diferencial de empleo, esta Comunidad empeora su posición relativá en todos los restantes indicadores. (Gráfico 13).

Es esta una Comunidad en la que, salvo el diferencial de paro que permanec inalterable, mejoran considerablemente todos los indicadores. (Gráfico 14)

Empeora considerablemente el diferencial de empleo, manteniendo inalterables el resto de los indicadores. (Gráfico 15).

GRÁFICO 10

EVOLUCIÓN DE LA MEJORA EXPERIMENTADA POR CASTILLA-LA MANCHA. (Elaboración propia)

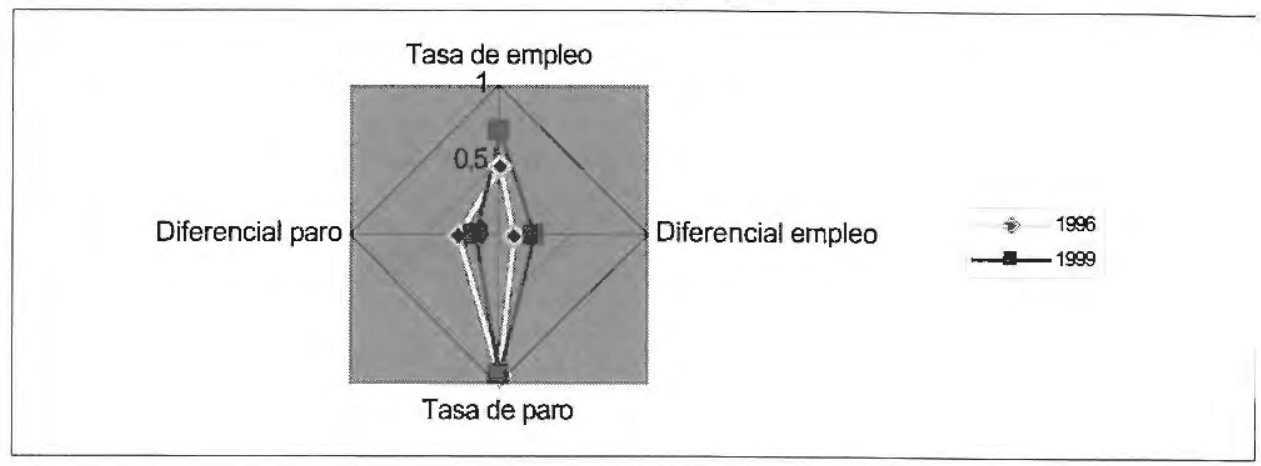

GRÁFICO I1

EVOLUCIÓN DE LA MEJORA EXPERIMENTADA POR LA COM. VALENCIANA. (Elaboración propia)

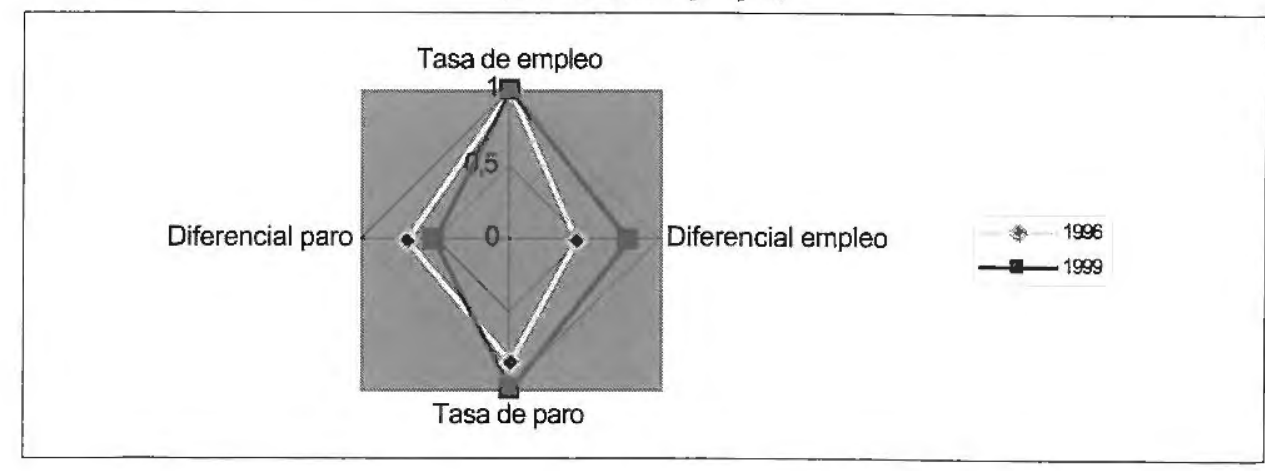




\section{GRÁFICO 12 \\ EVOLUCIÓN DE LA MEJORA EXPERIMENTADA POR EXTREMADURA. \\ (Elaboración propia)}

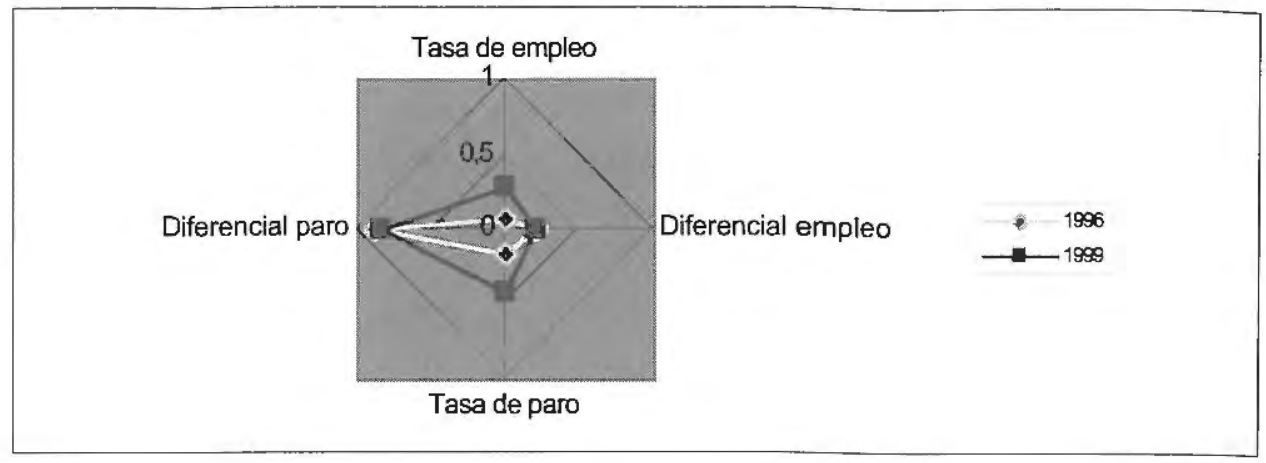

\section{GRÁFICO 13}

EVOLUCIÓN DE LA MEJORA EXPERIMENTADA POR GALICIA.

(Elaboración propia)

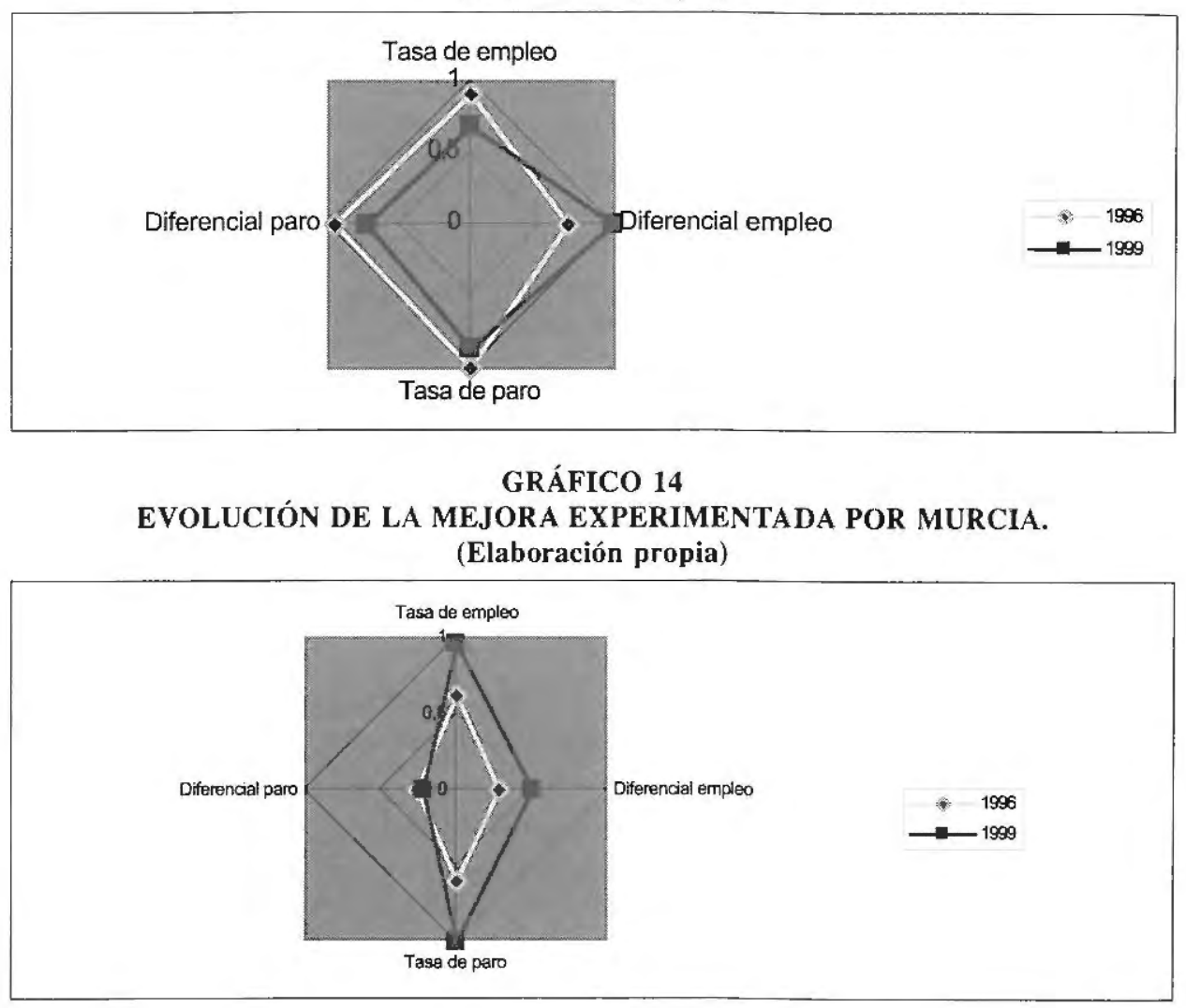




\section{GRÁFICO 15 \\ EVOLUCIÓN DE LA MEJORA EXPERIMENTADA POR CEUTA Y MELILLA. (Elaboración propia)}

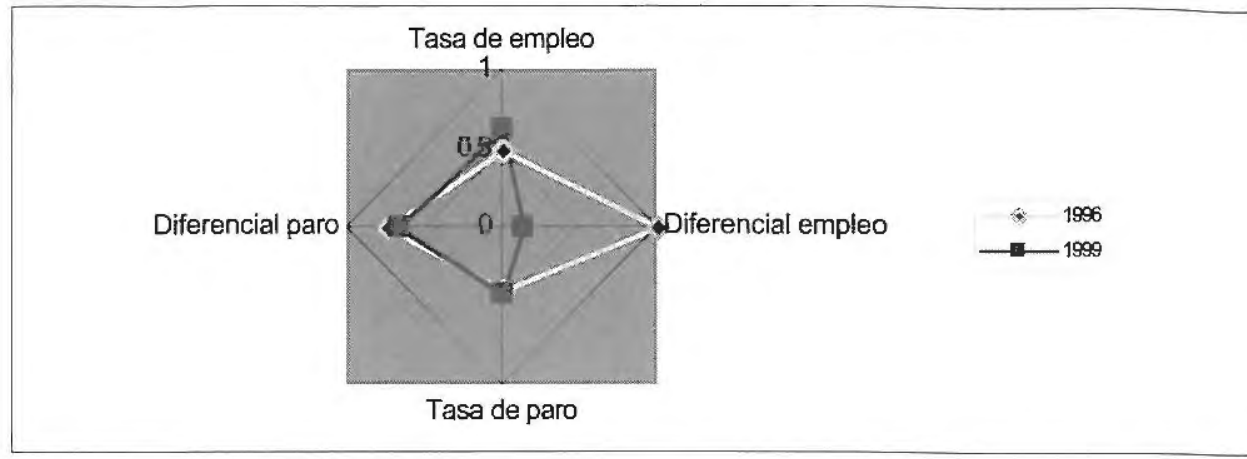

En el gráfico 16 mostramos la mejora global experimentada por cada Autono. mía utilizando el indicador conjunto.

De este gráfico se desprende que las Comunidades de Andalucía, Asturias ambas Castillas, Valencia, Extremadura y Murcia han evolucionada favorablement en sus direcciones de mejora. Mientras que han empeorado Cantabria, Galicia s Ceuta y Melilla. Con ello no queremos significar que las primeras sean las mejos situadas, pues, no olvidemos que esta técnica establece sólo relaciones de mejor y no posiciones absolutas. Además, y debido a los inconvenientes de la técnič empleada, a los que hicimos referencia en el apartado 2, no podemos aventuras acerca de las razones que llevan a una Comunidad a mejorar más o menos de Ic que lo hacen otras.

Lo que sí vuelve a ponerse de manifiesto, por otra parte, es la disparidad de comportamiento entre las regiones que la Unión Europea ha considerado en un mismo grupo. Dado que, como se establece en el propio Documento de clasificación, una de las bases de la agrupación es la mejora del empleo femenino, parece que la elección de las Comunidades que forman parte del mismo grupo no es la más adecuada, ya que no responden a los mismos estímulos y, en este sentido se pondría pensar en una agrupación altemativa.

El gráfico 17 se complementa con el del porcentaje de cambio, donde se representa el cambio porcentual en el indicador conjunto de mejora, experimentado del año 1996 al año 1999.

En atención a este porcentaje de cambio enumeramos las Comunidades en orden a una evolución más positiva. (Cuadro V).

Andalucía ocupa el primer lugar de las diez Comunidades consideradas, podemos, pues, considerar que nuestra región destaca positivamente dentro del grupo en que se ha visto encuadrada por la Unión Europea. Los esfuerzos que se están 


\section{GRÁFICO 16 \\ COMPARACIÓN DE ANDALUCÍA CON OTRAS COMUNIDADES. \\ INDICADOR CONJUNTO. (Elaboración propia)}

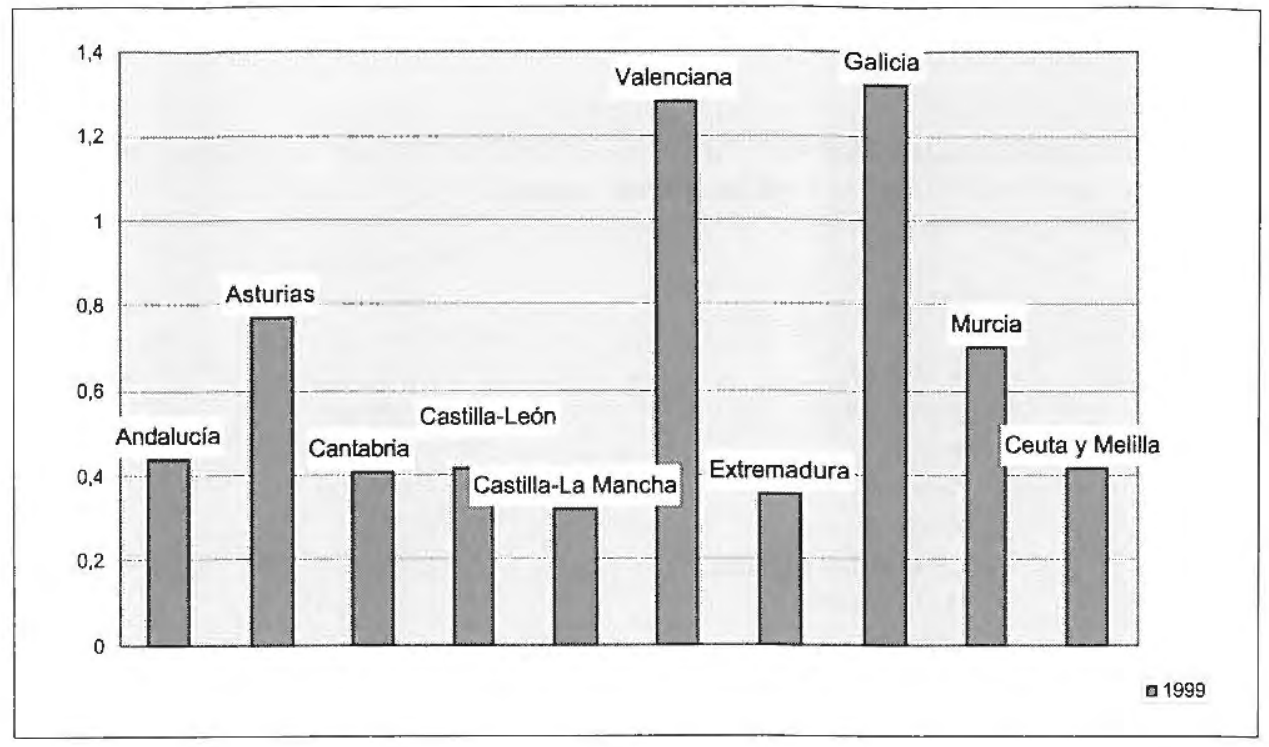

\section{GRÁFICO 17 \\ PORCENTAJE DE CAMBIO EN EL INDICADOR CONJUNTO.} (Elaboración propia)

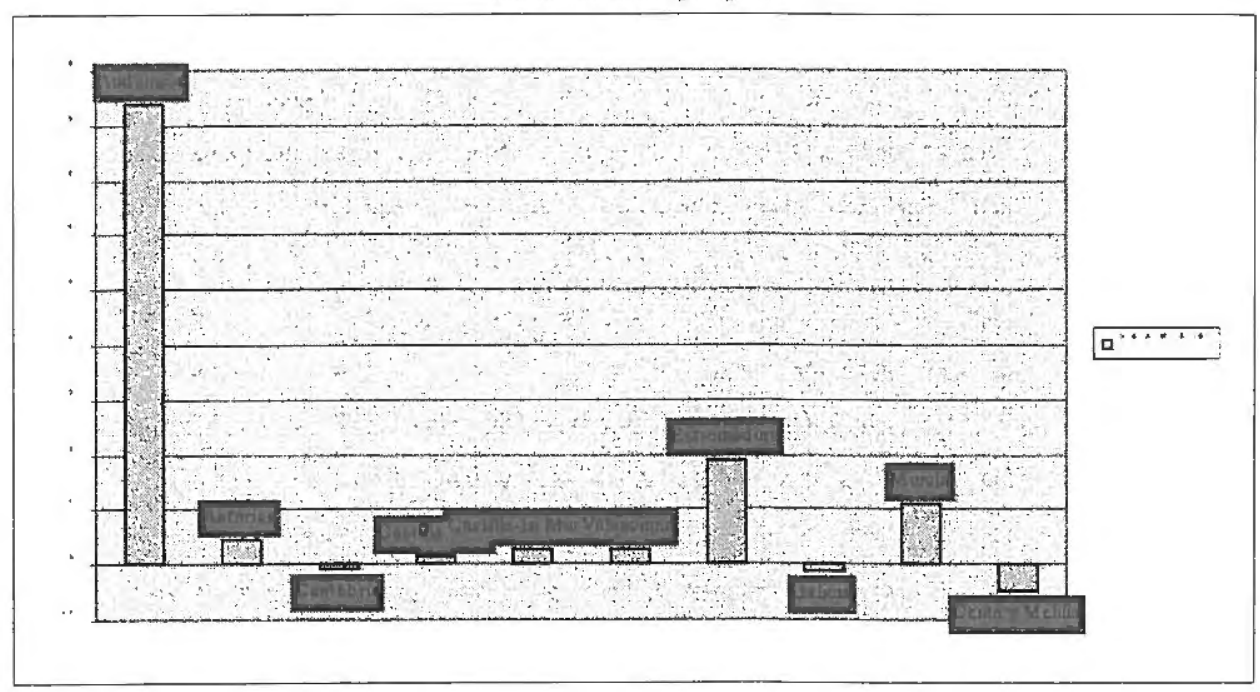


realizando en la misma están siendo, por tanto, recogidos por el mercado y están ayudando a una mejor posición relativa femenina frente a la masculina. Esto debe ser un acicate más para continuar por este camino.

\section{CUADRO V}

COMUNIDADES ORDENADAS SEGÚN SU PORCENTAJE DE CAMBIO. 1996-99. (Elaboración propia)

\begin{tabular}{lc}
\hline CCAA & \% Cambio \\
& $1996 / 1999$ \\
\hline Andalucía & 8,401742 \\
Extremadura & 1,903245 \\
Murcia & 1,088611 \\
Asturias & 0,433509 \\
Valenciana & 0,26679 \\
Castilla-La Mancha & 0,259435 \\
Castilla-León & 0,135163 \\
Cantabria & $-0,091709$ \\
Galicia & $-0,143614$ \\
Ceuta y Melilla & $-0,454144$ \\
\hline
\end{tabular}

\section{CONCLUSIONES}

En este trabajo hemos analizado la situación respecto al diferencial de género en el empleo y en el paro en la Comunidad Autónoma de Andalucía y la hemos comparado con la relativa a las otras Comunidades españolas que la Unión Europea, en base al Tratado de Amsterdam, y con objeto de repartir con mayor eficiencia los fondos estructurales, en busca del objetivo primordial que es el empleo, ha englobado como «Regiones objetivo 1», desde dos ópticas diferenciadas.

En primer lugar hemos tratado la mejora relativa que se ha producido de 1996 a 1999. Con respecto a esto extraemos las siguientes conclusiones:

- En cuanto al paro, medido a través de la tasa de paro, Andalucía sigue siendo la Región más castigada. Junto a esto es una de las Comunidades que ha reducido su diferencial de paro.

- En el empleo, medido a través de la tasa de empleo, y en el diferencial de género en el empleo que muestran los datos en nuestra Comunidad, la posición relativa de Andalucía ha mejorado muy poco. En ambas variables la mejora es alrededor de un $32 \%$ de la mejora experimentada por la Comunidad que más lo ha hecho. 
- El indicador global sitúa a Andalucía en la quinta posición de mejora, con comportamiento similar a Castilla-León y a Ceuta y Melilla, y muy alejada de la Comunidad que más ha mejorado en estos años (Galicia).

En segundo lugar hemos analizado la evolución de la mejora representando en un mismo gráfico la experimentada de 1993 a 1996 y de 1996 a 1999. Con respecto a esto concluimos lo siguiente:

- De 1996 a 1999 el empleo evoluciona en esta región frente a las otras mejor que como lo hizo de 1993 a 1996. No así el diferencial de género en el empleo, en el que prácticamente mantiene su posición.

- El diferencial de paro no se mueve, manteniendo la mayor de las mejoras que se producen en el resto de las Comunidades Autónomas. El paro mejora aunque no alcanza ni el $50 \%$ de la mejora que experimenta en el resto de las Comunidades.

- El indicador global nos muestra a Andalucía como la Comunidad que mejor porcentaje de cambio experimenta entre 1996 y 1999. Esto nos indica que evoluciona favorablemente y además mantiene dicha evolución en el tiempo. De aquí concluimos que las medidas de fomento de empleo femenino y lucha contra el paro de dicho colectivo, están dando más frutos que en el resto de las Comunidades con las que nos ha emparejado la Unión Europea. El camino seguido es, pues, adecuado.

\section{BIBLIOGRAFÍA}

ALCAIDE INCHAUSTI, J. El crecimiento económico desde la óptica regional. Cuademos de Información Económica, n 120-121, pp. 16-28, 1997.

ÁLVAREZ, J.A.; DÍAZ, F.M.; JIMÉNEZ, V. Mercados regionales de trabajo y desarrollo económico regional en España. Estudios de Economía Aplicada n 10, 1998.

GONZÁLEZ CALBERT, L. El mercado laboral en 1998: Logros y retos pendientes. Cuademos de Información Económica, ${ }^{\circ}$ 143, Febrero 1999.

JIMENO, J.F. Políticas de empleo para el nuevo milenio. Universidad de Alcalá, Fedea, Julio, 1999.

ENCUESTA DE COYUNTURA LABORAL. Ministerio de Trabajo y Asuntos Sociales. Centro de Publicaciones del Ministerio de Trabajo y Asuntos Sociales.

ENCUESTA DE POBLACIÓN ACTIVA. Instituto Nacional de Estadística.

ESTADÍSTICAS LABORALES. Instituto Nacional de Empleo.

MERCADO DE TRABAJO. Coyuntura Económica de Andalucía, n 33, 1998.

MOSLEY, H.; MAYER, A. Benohmaking National Labour Market. Performance: A radar Chart Approach. European Commission, Directorate-General V, Employment, Industrial Relations and Social Affairs, December 1998.

OBSERVATORIO DE RELACIONES INDUSTRIALES. Consejo Económico y Social. $\mathrm{N}^{\circ} 23$, 1999. 